\title{
First-principles studies of the electronic structure and magnetism in fayalites: $\mathrm{M}_{2} \mathrm{SiO}_{4}(\mathrm{M}=\mathrm{Fe}$ and $\mathrm{Co})$
}

\author{
Xuefan Jiang ${ }^{\mathrm{a}, \mathrm{b}}$, G.Y. Guo ${ }^{\mathrm{a}, \mathrm{c}, *}$ \\ a Department of Physics, National Taiwan University, Taipei 106, Taiwan \\ ${ }^{\mathrm{b}}$ Department of Physics, Changshu Institute of Technology, Changshu 215500, China \\ ${ }^{\mathrm{c}}$ National Synchrotron Radiation Research Center, Hsinchu 300, Taiwan
}

Available online 3 May 2004

\begin{abstract}
The electronic structure and magnetism in fayalites $\mathrm{M}_{2} \mathrm{SiO}_{4}(\mathrm{M}=\mathrm{Fe}$ and $\mathrm{Co})$ have been studied by density functional theory within the generalized gradient approximation with the on-site Coulomb energy taken into account (GGA $+\mathrm{U}$ ). Stable insulating antiferromagnetic solutions are obtained. It is found that the gap opening in these fayalites results mainly from strong on-site Coulomb interactions of the magnetic atoms. In the band structures, the conduction bands originate mainly from the $\mathrm{M}(3 \mathrm{~d})$ orbitals of $\mathrm{M}_{2} \mathrm{SiO}_{4}$. The top of valence bands consists of the $\mathrm{Fe}(3 \mathrm{~d}$ ) orbitals in $\mathrm{Fe}_{2} \mathrm{SiO}_{4}$, and of the $\mathrm{Co}(3 \mathrm{~d})-\mathrm{O}(2 \mathrm{p})$ hybridization in $\mathrm{Co}_{2} \mathrm{SiO}_{4}$. All calculated orbital magnetic moments are very small. (C) 2004 Elsevier B.V. All rights reserved.
\end{abstract}

PACS: 71.15.Mb; 75.30.Et; 91.60.Ed

Keywords: Fayalite; Electronic structure; Magnetism; Density functional theory

The naturally occurring olivine-type minerals form a wide range of solid solutions. Their crystal structure is characterized by isolated $\mathrm{SiO}_{4}$ tetrahedra and two octahedral cation sites. Fayalites, $\mathrm{M}_{2} \mathrm{SiO}_{4}(\mathrm{M}=\mathrm{Fe}$ and $\mathrm{Co})$, are the end-member of the olivine-type silicate that has an orthorhombic crystal structure with the space group Pnma [1]. There are two crystallographically inequivalent $\mathrm{M}$ sites, where M1 (4a) ions are sites of inversion symmetry and M2 (4c) ions are in the plane of

\footnotetext{
*Corresponding author. Department of Physics, National Taiwan University, 1 Sec. 4, Roosevelt Road, Taipei 106, Taiwan. Tel.: + 886-2-23626937; fax: + 886-2-23639984.

E-mail address: gyguo@phys.ntu.edu.tw (G.Y. Guo).
}

mirror symmetry. In both cases the magnetic moments on M2 sites are parallel to one of the crystallographic b-axis. The M1 moments, on the other hand, are parallel to none of three principal axes and their directions show strong temperature dependence [2]. These behaviors could stem from strong correlations among the crystal structure and the electronic or magnetic properties. At room temperature and pressure, fayalite is an insulator $[3,4]$.

Recently, the electronic structure has been studied by experimental investigation for better understanding of its properties [4]. In particular, the application of ab initio techniques to the study of systems of geophysical interest has expanded 
considerably and even detailed studies of the thermodynamical properties of some systems are available $[5,6]$. The structural, electronic and magnetic properties of $\mathrm{Fe}_{2} \mathrm{SiO}_{4}$ fayalite were determined [7] by using the density functional theory (DFT) in a plane-wave pseudopotential framework with generalized gradient approximation (GGA) [10]. However, their electronic structure calculations within the GGA predicted a metallic ground state, contrary to experimental evidence that indicates an insulating, possibly Mott-Hubbard, behavior at ambient pressure and temperature.

The purpose of the present investigation is to provide a more accurate theoretical understanding of the electronic and magnetic properties of the $\mathrm{M}_{2} \mathrm{SiO}_{4}(\mathrm{M}=\mathrm{Fe}$ and $\mathrm{Co})$ fayalites by using the full-potential projected augmented-wave (PAW) method [8] as implemented in the Vienna ab initio simulation package (VASP) [9]. The calculations are based on first-principles DFT within GGA with the on-site Coulomb interactions taken into account, the so-called GGA + U method [11]. The values of $U=4.5$ [7] (6.1 [12]) eV and $J=0.90$ $(0.92) \mathrm{eV}$ [12] for $\mathrm{Fe}^{2+}\left(\mathrm{Co}^{2+}\right)$ in $\mathrm{M}_{2} \mathrm{SiO}_{4}(\mathrm{M}=\mathrm{Fe}$ and $\mathrm{Co}$ ) are used in the following spin polarized GGA + U calculations. The density of states (DOS) and partial DOS were calculated by the linear tetrahedron method [12]. The plane-wave cut-off energy of $400 \mathrm{eV}$ and the convergence criteria for energy of $10^{-4} \mathrm{eV}$ are selected. The Monkhorst-pack $k$-points generation scheme is used with a grid of $3 \times 5 \times 7$ points in the irreducible Brillouin zone (IBZ) for an orthorhombic cell, which means that the number of the $k$-points in the IBZ used in self-consistent calculation is 24 .

The magnetic structures of $\mathrm{Fe}_{2} \mathrm{SiO}_{4}$ and $\mathrm{Co}_{2}$ $\mathrm{SiO}_{4}$ are essentially similar [2]. $\mathrm{M}_{2} \mathrm{SiO}_{4}(\mathrm{M}=\mathrm{Fe}$ and $\mathrm{Co}$ ) fayalites are known to be an antiferromagnetic (AF) compound with slightly noncollinear arrangement of spin on M1 site, but this noncollinearity will not be addressed in the present calculations for simplicity. In the spin configuration the AF interaction between corner-sharing octahedral is selected [2]. The optimal lattice constants and internal structural parameters from the GGA + U calculations are used.
Contrary to the previous GGA result of $\mathrm{Fe}_{2} \mathrm{SiO}_{4}$ [7] that predicted a metallic ground state, present electronic band structure of $\mathrm{Fe}_{2} \mathrm{SiO}_{4}$ from the GGA $+\mathrm{U}$ shows the insulating behavior with an indirect gap of $1.49 \mathrm{eV}$ opened between the $\mathrm{Z}$ and the $\Gamma$ points. It shows a very small dispersion of the top most valence band in the $\Gamma \mathrm{Z}$ direction parallel to the c-axis, but a rather flat along other directions perpendicular to the $c$-axis in the ГXSY plane. The experimental band-edge absorption [13] is not so distinct, because an absorption threshold exhibits a tail into the optical gap region. It seems that the experimental results support our findings that $\mathrm{Fe}_{2} \mathrm{SiO}_{4}$ is an indirect gap material. The $\mathrm{GGA}+\mathrm{U}$ results lead us to the conclusion that electronic correlations of the Hubbard type are important for the proper gap opening in the $\mathrm{Fe}_{2} \mathrm{SiO}_{4}$ fayalite.

In the electronic structure of $\mathrm{Fe}_{2} \mathrm{SiO}_{4}$ from the GGA + U calculations the Si-projected DOS component in the valence-band energy range is very small. The $\mathrm{O}$-atom orbital weight is concentrated mainly in the region of energy smaller than $1.36 \mathrm{eV}$ below the top of valence bands, so that $\mathrm{Fe}(3 \mathrm{~d})$ $\mathrm{O}(2 \mathrm{p})$ hybridization effects should appear mainly in the lower valence-band energy range. This socalled split-off band comprises four single bands, in which two higher energy and two lower energy bands originate mainly from the $\mathrm{Fe} 2(3 \mathrm{~d})$ and the Fe1(3d) orbitals, respectively. The broad lower energy valence bands starting from $1.36 \mathrm{eV}$ below the top of valence bands are constituted from the $\mathrm{Fe}(3 \mathrm{~d})-\mathrm{O}(2 \mathrm{p})$ hybridization. The conduction bands originate mainly from the $\mathrm{Fe}(3 \mathrm{~d})$ orbitals, where the lower energy conduction bands below about $2.5 \mathrm{eV}$ are attributed mainly to the $\mathrm{Fe} 1\left(\mathrm{t}_{2 \mathrm{~g}}\right)$ orbitals. The procedures will be described in detail elsewhere [14].

In Fig. 1 we report the GGA calculated electronic energy bands of $\mathrm{Co}_{2} \mathrm{SiO}_{4}$ around Fermi energy. The electronic energy band structure is marked by the flatness of the bands around the Fermi level along [0 001$]$ ( $\mathrm{ZU}, \mathrm{RT})$ directions and the relatively large dispersion in the $\left[\begin{array}{lll}0 & 1 & 0\end{array}\right]$ direction $(\Gamma Y, X S, Z T$, UR). This behavior is similar to that of $\mathrm{Fe}_{2} \mathrm{SiO}_{4}$ from the previous GGA calculations [7]. The GGA band structure of $\mathrm{Co}_{2} \mathrm{SiO}_{4}$ appears to be a band 


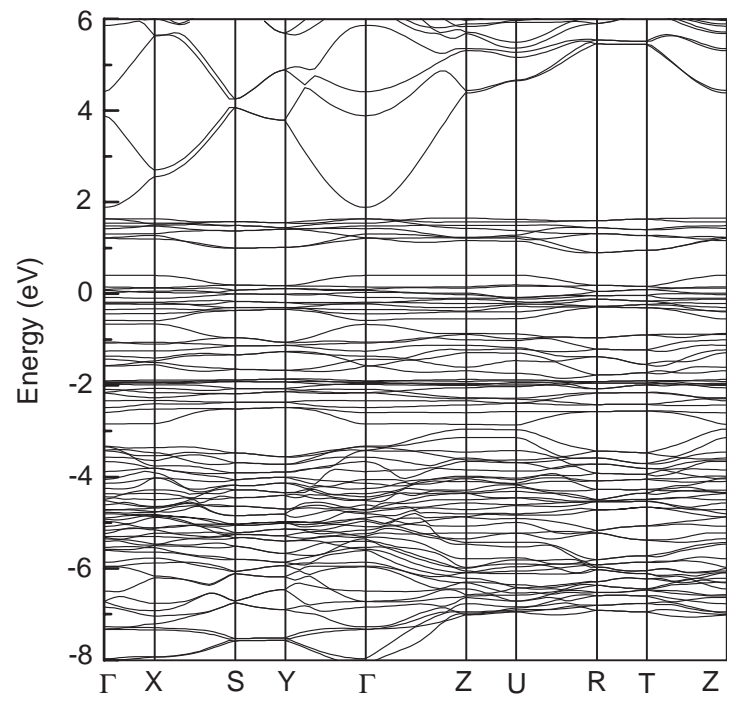

Fig. 1. The electronic energy bands of $\mathrm{Co}_{2} \mathrm{SiO}_{4}$ from the GGA calculations. The zero energy denotes the Fermi level.

metal with two cobalt d-bands crossing the Fermi level, in contrast to the experimental evidence of insulator [15], indicating the importance of strong electron correlation effects. In Fig. 2(a), we show an accurate GGA + U calculation of electronic energy bands with an indirect band gap of $3.13 \mathrm{eV}$ in $\mathrm{Co}_{2} \mathrm{SiO}_{4}$. This is consistent with the experimental value of several eV [15]. From the DOS (Fig. 2(b)) of $\mathrm{Co}_{2} \mathrm{SiO}_{4}$ it is shown that all valence bands of $\mathrm{Co}_{2} \mathrm{SiO}_{4}$ are formed by $\mathrm{Co}(3 \mathrm{~d})-\mathrm{O}(2 \mathrm{p})$ orbital states hybridization leading to the appearance of relatively strong dispersion along the $b$ direction (Fig. 2(a)). The conduction bands are composed mainly of the $\mathrm{Co}(3 \mathrm{~d})$ orbitals. The Siprojected DOS component is very small.

The calculated spin and orbital magnetic moments from the GGA $+\mathrm{U}$ for $\mathrm{M}_{2} \mathrm{SiO}_{4}(\mathrm{M}=\mathrm{Fe}$ and Co) are listed in Table 1. Within the GGA + U, the spin moments of $3.65 \mu_{\mathrm{B}}$ per iron atom in $\mathrm{Fe}_{2} \mathrm{SiO}_{4}$ are found to be slightly smaller than the experimental values of 4.40 and $4.41 \mu_{\mathrm{B}}$ for Fe1 and Fe2 at a temperature of about $10 \mathrm{~K}$ [2], respectively. At the same time, the present GGA + U calculation gives a rather small orbital moment of 0.044 and $0.024 \mu_{\mathrm{B}}$ for per $\mathrm{Fe} 1$ and $\mathrm{Fe} 2$ atoms, respectively. In spite of the orbital magnetic moment of $\mathrm{Fe}(3 \mathrm{~d})$
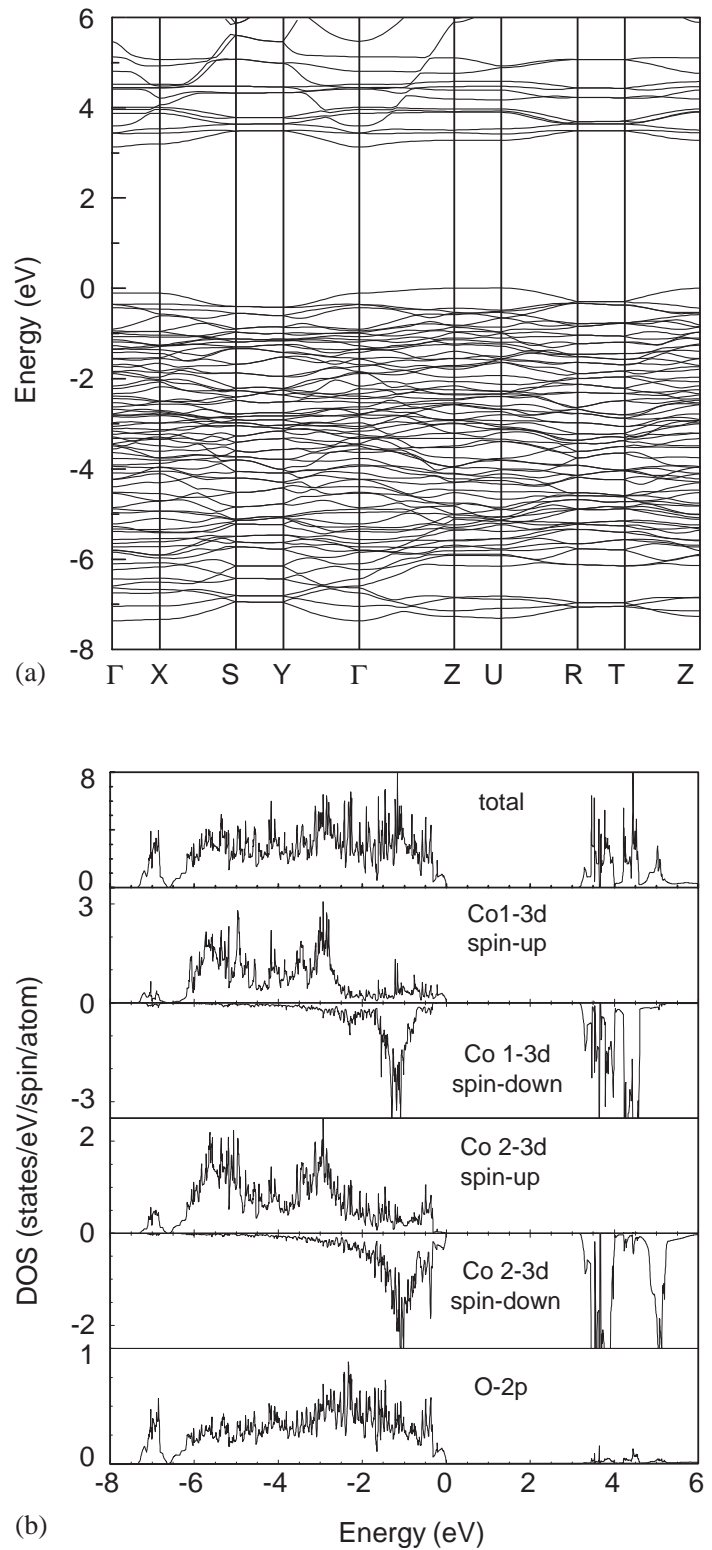

Fig. 2. The electronic structure of $\mathrm{Co}_{2} \mathrm{SiO}_{4}$ from the GGA + U calculations. (a) electronic energy bands; (b) total DOS per formula and partial DOS per atom and spin. The zero energy denotes the top of valence bands.

orbital having the same sign as the spin moment, indicating that the $3 \mathrm{~d}$ orbital is over half-filled in accordance with Hund's rule, our calculated values for the magnetic moment are still slightly smaller 
Table 1

Spin and orbital magnetic moments $\left(\mu_{\mathrm{B}} /\right.$ atom $)$ of iron and cobalt atoms from the GGA + U calculation

\begin{tabular}{|c|c|c|c|c|c|}
\hline & & \multicolumn{2}{|l|}{ Spin } & \multicolumn{2}{|c|}{ Orbital } \\
\hline & & M1 & M2 & M1 & M2 \\
\hline \multirow[t]{2}{*}{$\mathrm{Fe}_{2} \mathrm{SiO}_{4}$} & $\mathrm{GGA}+\mathrm{U}$ & 3.65 & 3.65 & \multirow[t]{2}{*}{0.044} & \multirow[t]{2}{*}{0.024} \\
\hline & Expt. [2] & 4.40 & 4.41 & & \\
\hline \multirow[t]{2}{*}{$\mathrm{Co}_{2} \mathrm{SiO}_{4}$} & $\mathrm{GGA}+\mathrm{U}$ & 2.77 & 2.78 & \multirow[t]{2}{*}{0.046} & \multirow[t]{2}{*}{0.044} \\
\hline & Expt. [2] & 3.90 & 3.64 & & \\
\hline
\end{tabular}

than experimental measurements. In $\mathrm{Co}_{2} \mathrm{SiO}_{4}$, the spin moments of $\mathrm{Co}$ are smaller than those of $\mathrm{Fe}_{2} \mathrm{SiO}_{4}$ due to the valence configuration $\mathrm{Co}^{2+}$ $\left(3 \mathrm{~d}^{7}\right)$ of three spin-only electrons. The calculated results are also slightly smaller than the experimental value [2] as seen in Table 1 . The very small orbital magnetic moments in $\mathrm{Co}_{2} \mathrm{SiO}_{4}$ are similar to those of $\mathrm{Fe}_{2} \mathrm{SiO}_{4}$. In fact, for $\mathrm{M}_{2} \mathrm{SiO}_{4}(\mathrm{M}=\mathrm{Fe}$ and $\mathrm{Co}$ ), in the fourfold M1 position the orientations of the moments are canted away from the corresponding crystallographic axes for $T \rightarrow 0 \mathrm{~K}$ [2]. The magnetic structure of $\mathrm{M}_{2} \mathrm{SiO}_{4}$ is complex, so that for the magnitudes and directions of the magnetic moments the difference between calculation and experiment might result from the fact that the simple collinear magnetic structures were considered in the present and in previous calculations. For $\mathrm{Si}(3 \mathrm{~s})$ and $\mathrm{O}(2 \mathrm{p})$ orbitals, the orbital moments are negligibly small.
The authors are grateful to the support from the National Science Council of Taiwan under Grant No. NSC91-2816-M-002-0009-6. X.F.J. was supported in part by the Natural Science Foundation of Jiangsu Department of Education, China.

\section{References}

[1] R.W.G. Wyckoff, Crystal Structures, Vol. 3, 2nd Edition, Krieger, FL, 1981, b10 (Chapter VIII).

[2] H. Fuess, O. Ballet, W. Lottermoser, in: S. Ghose, J.M.D. Coey, E. Salje (Eds.), Structural and Magnetic Phase Transitions in Minerals, Springer, Berlin, 1988.

[3] H.K. Mao, P.M. Bell, Science 176 (1972) 403.

[4] Q. Williams, et al., J. Geophys. Res. 95 (1990) 21549.

[5] B.B. Karki, R.M. Wentzcovitch, S. de Gironcoli, S. Baroni, Science 286 (1999) 1705.

[6] D. Alfè, M.J. Gillan, G.D. Price, Nature (London) 401 (1999) 462;

D. Alfè, M.J. Gillan, G.D. Price, Nature (London) 405 (2000) 172.

[7] M. Cococcioni, A.D. Corso, S. de Gironcoli, Phys. Rev. B 67 (2003) 094106.

[8] P.E. Blöchl, Phys. Rev. B 50 (1994) 17953.

[9] G. Kresse, J. Turthmueller, Phys. Rev. B 54 (1996) 11169.

[10] J.P. Perdew, K. Burke, M. Ernzerhof, Phys. Rev. Lett. 71 (1996) 3865.

[11] V.I. Anisimov, F. Aryasetiawan, A.I. Lichtenstein, J. Phys.: Condens. Matter 9 (1997) 767.

[12] I.V. Solovyev, P.H. Dederichs, V.I. Anisimov, Phys. Rev. B 50 (1994) 16861.

[13] H.G. Smith, K. Langer, Am. Mineral. 67 (1982) 343.

[14] X.F. Jiang, G.Y. Guo, Phys. Rev. B 69 (2004) 155108.

[15] T.J. Shankland, Phys. Earth Planet Inter. 10 (1975) 209. 